\title{
O XENODIAGNÓSTICO NA DOENÇA DE CHAGAS: INFLUÊNCIA DO SEXO DOS TRIATOMÍNEOS
}

\author{
Elisabeth Visone Nunes, Rubens Campos, Carmem do Socorro Guilherme, \\ José Eduardo Tolezano, Antônio Augusto Baillot Moreira, Hertha Bárbara \\ Wüllert Souza, Clóvis Kiomitsu Takiguti e Vicente Amato Neto
}

\begin{abstract}
Foi avaliado, comparativamente, o desempenho de fêmeas e machos de Triatoma infestans, em $5^{\circ}$ estádio de desenvolvimento, em xenodiagnósticos para a tripanossomíase americana. Estes exames foram realizados em 40 pacientes, em fase crônica da doença de Chagas. Em cada exame utilizou-se 20 ninfas fêmeas e 20 ninfas machos, que foram pesadas imediatamente antes e após o repasto. $O$ exame individual do conteudo intestinal dos triatomíneos foi efetuado aos 20,30,60 e 90 dias após a aplicação dos xenodiagnósticos. As ninfas fêmeas e machos, ingeriram, em média, 230 e $210 \mathrm{mg}$ de sangue, respectivamente. Os resultados revelaram 15 (37,5\%) xenodiagnósticos positivos; todavia, em nenhum destes exames foi verificada positivação, para $\mathrm{T}$. cruzi, de todas as ninfas utilizadas. As ninfas fêmeas ingeriram significalivamente mais sangue que as ninfas machos, isto vem de encontro com as observações de maior positividade, para T. cruzi entre as fêmeas, sugerindo, serem estas ninfas capazes de possibilitar maior incremento à sensibilidade do xenodiagnóstico na fase crônica da doença de Chagas, sendo necessários novos estudos para confirmação desta hipótese.
\end{abstract}

Palavras-chaves: Xenodiagnóstico. Doença de Chagas. Sensibilidade do xenodiagnóstico. Tripanossomíase americana.

Desde que Brumpt, em 1914, mostrou a possibilidade de utilização de xenodiagnóstico para o diagnóstico de moléstias parasitárias e, em particular, da doença de Chagas, por intermédio do próprio vetor, diversos estudos têm sido realizados na tentativa de demonstrar ${ }^{7813141516}$ ou

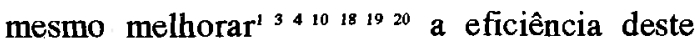
procedimento laboratorial.

Essa técnica, a que Brumpt ${ }^{2}$ chamou de cultura natural em hospedeiro favorável, é a de maior utilização para o diagnóstico parasitológico da tripanossomíase americana, na fase crônica, apesar dos incovenientes de positividade variável, demora de tempo para liberação do resultado, necessidade de contato do triatomíneo com paciente $e$ necessidade de criação destes insetos em laboratório.

\footnotetext{
Trabalho da Seção de Parasitoses Sistêmicas, Instituto Adolfo Lutz e do Laboratório de Investigaçāo Médica e Departamento de Medicina Preventiva da Faculdade de Medicina, Universidade de São Paulo, São Paulo, SP. Endereço para correspondência: Prof. José Eduardo Tolezano. Instituto Adolfo Lutz. Av. Dr. Arnaldo 351, $10^{\circ}$ andar, Cerqueira Cesar, 01246 São Paulo, SP, Brasil. Recebido para publicação em 24/06/91.
}

No que tange aos barbeiros, essenciais para a realização do xenodiagnóstico, desde os primeiros estudos sobre a biologia destes hemípteros, criados em laboratório, é conhecido o fato das fêmeas serem capazes de ingerir maiores volumes de sangue, em um único repasto sanguíneo, que os machos.

No presente estudo, os autores objetivaram avaliar comparativamente o desempenho de ninfas fềmeas e machos de Triatoma infestans, em $5^{\circ}$ estádio de desenvolvimento, aplicados em pacientes com doença de Chagas, todos em fase crônica.

\section{MATERIAL E MÉTODOS}

Foram realizados xenodiagnósticos em um total de 40 pacientes, todos em fase crônica da doença de Chagas. Em cada paciente foi aplicado um único xenodiagnóstico utilizando-se 40 ninfas de $5^{\circ}$ estádio de Triatoma infestans, sendo 20 ninfas fêmeas e 20 ninfas machos.

A diferenciação sexual em ninfas fêmeas e ninfas machos, foi realizada levando-se em consideração as características morfológicas do 
Nunes EV, Campos R, Guilherme CS, Tolezano JE, Moreira AAB, Souza HBW, Takiguti CK, Amato-Neto V. $O$ xenodiagnóstico na doença de Chagas: influência do sexo dos triatomíneos. Revista da Sociedade Brasileira de Medicina Tropical 24:245-250, out-dez, 1991

oitavo e do nono esternito destes artrópodes ${ }^{911}$.

Os insetos foram pesados, segundo sexo, imediatamente antes e após o repasto sanguineo, para avaliação da quantidade de sangue ingerida e o respectivo ganho de peso.

Após a realização do xenodiagnóstico, as ninfas foram mantidas em jejum e seu conteúdo intestinal foi examinado, individualmente, aos $20,30,60 \mathrm{e}$ 90 dias.

A avaliação estatística dos resultados observados, em relação ao peso dos grupos de triatomíneos fêmeas e machos, foi realizada pela utilização do teste " $t$ " de Student ao nivel de $\mathrm{P}<0,05$. Para avaliação da positividade para $T$. cruzi, obtida nos dois grupos de "barbeiros", foi aplicado o teste do qui quadrado com utilização do coeficiente de correção de Yates para $\underline{\mathrm{P}}<0,05$.

\section{RESULTADOS}

Dos 40 xenodiagnósticos realizados observaram-se $15(37,5 \%)$ positivos para $T$. cruzi.

Pelos resultados ao exame do conteúdo intestinal dos triatomíneos examinados, verificou-se maior positivação entre as ninfas fêmeas (Tabela 1).

Nos 15 exames positivos, encontrou-se positividade de $29,1 \%$ e $24,7 \%$, respectivamente para exemplares fêmeas e machos, nos quais foram efetuados os quatro exames do conteúdo intestinal. Embora tenham sido as ninfas fêmeas aquelas que se infectaram em maior número, o teste do qui quadrado indicou serem não significativas, ao nivel de $\underline{\mathbf{P}}<0,05$, as diferenças observadas.

$\mathrm{O}$ número de ninfas positivas em cada um dos xenodiagnósticos positivos variou de 1 a 16 ninfas (Tabela 2).

A comparação entre as médias de peso de triatomíneos antes e após o repasto sanguíneo e a respectiva quantidade de sangue ingerida, indicou, sempre, maiores valores para as ninfas fêmeas (Figura 1).

Dentre as ninfas utilizadas nos 15 xenodiagnósticos positivos, observou-se quea média dos pesos anteriores e posteriores ao repasto foi, respectivamente de 77,5 e $314,7 \mathrm{mg}$ para as fêmeas e 71,4 e 278,1 mg para os machos (Figura 1).

A quantidade média de sangue ingerida, da ordem de $237,2 \mathrm{mg}$ para as fêmeas e $206,7 \mathrm{mg}$ para os machos, foi francamente favorável às fêmeas, com " $\mathrm{t}$ " $=3,25$, significativo ao nível de $\underline{\mathrm{P}}<0,05$.

Tabela 1 - Resultados observados ao exame do conteúdo intestinal dos triatomíneos, ao longo dos exames realizados*.

\begin{tabular}{|c|c|c|c|c|c|c|c|c|}
\hline \multirow{2}{*}{ Ninfas } & \multicolumn{2}{|c|}{$1^{\circ}$ Exame** } & \multicolumn{2}{|c|}{$2^{\circ}$ Exame*** } & \multicolumn{2}{|c|}{$3^{\circ}$ Exame $^{* * * *}$} & \multicolumn{2}{|c|}{$4^{\circ}$ Exame $* * * * * *$} \\
\hline & fêmeas & machos & fềmeas & machos & fêmeas & machos & fêmeas & machos \\
\hline Positivas & 30 & 14 & 44 & 32 & 50 & 37 & 51 & 38 \\
\hline Negativas & 701 & 734 & 603 & 657 & 461 & 462 & 327 & 328 \\
\hline Mortas & 69 & 52 & 153 & 111 & 289 & 301 & 422 & 434 \\
\hline Total & 800 & 800 & 800 & 800 & 800 & 800 & 800 & 800 \\
\hline
\end{tabular}

* Os resultados positivos e a mortalidade são cumulativos

** 20 dias após a aplicação do xenodiagnóstico

*** 30 dias após a aplicação do xenodiagnóstico

**** 60 dias após a aplicação do xenodiagnóstico

***** 90 dias após a aplicação do xenodiagnóstico 
Nunes EV, Campos R, Guilherme CS, Tolezano JE, Moreira AAB, Souza HBW, Takiguti CK, Amato-Neto V. O xenodiagnóstico na doença de Chagas: influência do sexo dos triatomíneos. Revista da Sociedade Brasileira de Medicina Tropical 24:245-250, out-dez, 1991

Tabela 2 - Resultados verificados após os quatro exames do conteúdo intestinal das ninfas utilizadas nos 15 xenodiagnósticos positivos.

Pacientes $\frac{\text { Ninfas positivas }}{\text { fềmeas machos }} \quad \frac{\text { Ninfas negativas }}{\text { fêmeas machos }} \quad \frac{\text { Ninfas mortas* }}{\text { fềmeas machos }} \quad \frac{}{\text { fềmeas machos }}$

M.J.A.

$7 \quad 3$

30

10

17

20

20

S.P.L.

6

8

$5 \quad 8$

9

4

20

20

M.Z.S.

$$
6
$$

4

$9 \quad 11$

5

5

20

20

A.M.L.

5

$8 \quad 6$

7

13

20

20

M.C.V.

21

12

12

6

7

20

20

Q.S.

$0 \quad 1$

$9 \quad 9$

11

10

20

20

J.A.S.

2

0

$\begin{array}{ll}6 & 7\end{array}$

12

13

20

20

M.V.R.F.

02

23

18

15

20

20

H.M.

$$
5
$$

3

11

$14 \quad 16$

20

20

o.c.

$0 \quad 1$

94

$11 \quad 15$

20

20

J.B.S.

$$
4
$$

4

$7 \quad 6$

$9 \quad 10$

20

20

J.N.D.C.

10

6

84

$2 \quad 10$

20

20

J.A.B.

2

$16 \quad 16$

2

4

20

20

A.M.S.

11

$15 \quad 16$

4

3

20

20

J.T.S.

1

14

13

54

20

20

* Ninfas mortas antes que fosse possível a realização de um ou mais dos quatro exames do conteúdo intestinal.

Deve ser mencionado, também, que nos outros 25 xenodiagnósticos negativos, a média de peso antes e após a alimentação sanguínea no paciente foi, respectivamente de $83,3 \mathrm{e}$ $306,1 \mathrm{mg}$ para as fêmeas e $76,8 \mathrm{e} 291,1 \mathrm{mg}$ para os machos.

Tanto as ninfas fêmeas como as ninfas machos revelaram idêntica capacidade de distensão abdominal, ou seja, aumentaram, em média 3,8 vezes o seu peso após o repasto. 
Nunes EV, Campos R, Guilherme CS, Tolezano JE, Moreira AAB, Souza HBW, Takiguti CK, Amato-Neto V. O xenodiagnóstico na doença de Chagas: influência do sexo dos triatomíneos. Revista da Sociedade Brasileira de Medicina Tropical 24:245-250, out-dez, 1991

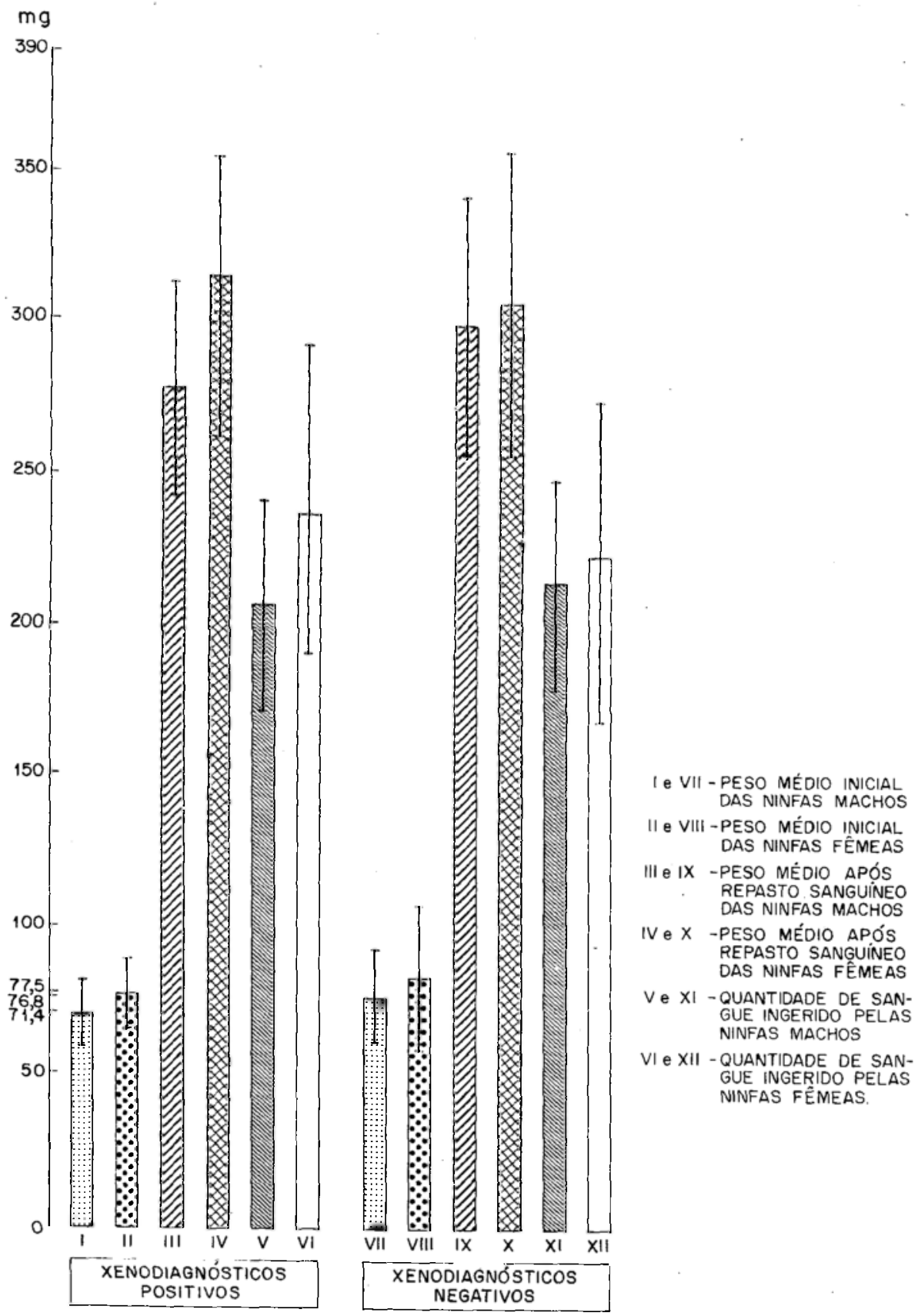

Figura 1 - Comparação entre as médias de peso e quantidade de sangue ingerido pelas ninfas de triatomíneos, segundo o sexo e resultados observados.

\section{DISCUSSÃO}

Estudos comparativos entre as técnicas parasitológicas da hemocultura e do xenodiagnóstico para doença de Chagas, realizadas por diversos pesquisadores, concluíram favoravelmente ao xenodiagnóstico ${ }^{3614}$.

De maneira geral, o xenodiagnóstico é considerado entre as melhores técnicas para diagnóstico parasitológico da tripanossomíase 
Nunes EV, Campos R, Guilherme CS, Tolezano JE, Moreira AAB, Souza HBW, Takiguti CK, Amato-Neto V. $O$ xenodiagnóstico na doença de Chagas: influência do sexo dos triatomíneos. Revista da Sociedade Brasileira de Medicina Tropical 24:245-250, out-dez, 1991

americana, em sua fase crônica, pelo fato de possibilitar exame de maior volume de sangue, ainda que de forma indireta, do que por outras técnicas de demonstração do Trypanosoma cruzi no sangue ${ }^{1719}$.

A verificação, no presente trabalho, de $37,5 \%$ de positividade nos xenodiagnósticos, aplicados em pacientes em fase crônica da infecção chagásica, não difere de observaçōes efetuadas por outros autores $^{4} 61316$.

A análise da distribuição dos resultados obtidos nos 40 xenodiagnósticos, ao longo de quatro exames do conteúdo instestinal dos triatomíneos (Tabela 1), veio confirmar maior positividade para T. cruzi entre ninfas fêmeas. Entretanto, o teste do qui quadrado não revelou diferenças estatisticamente significantes, ao nível de $\underline{\mathrm{P}}<0,05$, para a positividade para $T$. cruzi, entre as ninfas fêmeas e machos.

$\mathrm{Na}$ ocasião do quarto exame do conteúdo intestinal dos "barbeiros", a taxa de mortalidade ultrapassou a $50 \%$ tanto para o grupo das ninfas fêmeas como das ninfas machos.

A constatação de que em nenhum dos xenodiagnósticos positivos tivesse sido demonstrada a infecção de $100 \%$ das ninfas fêmeas e/ou machos (Tabela 2), vem confirmar hipótese de que na fase crônica da doença de Chagas, a contaminação, por $T$. cruzi, dos triatomíneos utilizados seria, também, função de "variável" relacionada com a casualidade do encontro de parasitas em número suficiente, naquele volume de sangue ingerido, quando da aplicação desta técnica parasitológica.

Embora tenha sido mostrado serem as fêmeas capazes de ingestão significativamente maior de sangue do que os machos, a capacidade de distensão abdominal resultante do repasto sanguíneo, foi semelhante nos dois grupos de ninfas, ou seja, revelaram aumento de 3,8 vezes seu peso inicial. Em última análise, a capacidade de distensão abdominal parece refletir volume total de sangue que poderá ser ingerido. Assim sendo, tendo os machos apresentado peso inicial menor do que as fêmeas, o tamanho do repasto sanguíneo acabou sendo, conseqüentemente, menor nos machos.

A alta taxa da mortalidade, verificada ao longo da investigação, pode ter exercido interferência no resultado final.
Entretanto, é patente que os efeitos do acaso intervirão para que uma ninfa de triatomíneo venha a ingerir $T$. cruzi um número su ficiente para se infectar durante o repasto sanguíneo, realizado num paciente em fase crônica da tripanossomíase americana.

Finalmente, a confirmação da capacidade de ingestão de maior quantidade de sangue e o maior número de insetos fêmeas infectadas por T. cruzi, indicam a necessidade de novos estudos, especialmente em situações em que a parasitemia da fonte infectante seja controlada.

\section{SUMMARY}

We evaluated, comparatively, the performance of female and male Triatoma infestans in the $5^{\text {th }}$ instar of development, in xenodiagnosis for Chagas 'disease. Xenodiagnosis were done in 40 patients with chronic infectans. For each diagnosis 20 nymphs female and 20 nymphs males were used, which were weighed immediately before and after the meal. Intestinal contents were examined about 20,30,60 and 90 days after the xenodiagnosis application. The females and males ingested the mean of 230 and $210 \mathrm{mg}$ of blood, respectively. The results revealed positivity of 15 (37.5\%) out 40 xenodiagnosis, but any of the positive tests show $100 \%$ of the nymphs, female and/or male infected by $\mathrm{T}$. cruzi. The females ingested significantly more blood than the males; this is coincidental with the higher female positivation to $\mathrm{T}$. cruzi here observed. Our results suggest that the female nymphs seem able to increase the sensibility of the xenodiagnosis for chronic patients with Chagas'disease being necessary further studies are necessary to confirm this hypothesis.

Key-words: Xenodiagnosis. Chagas'disease. Xenodiagnosis sensibility. American tripanosomiasis.

\section{REFERÊNCIAS BIBLIOGRÁFICAS}

1. Almeida SP, Sherlock IA, Fahel E. Novo procedimento de xenodiagnóstico na forma crônica da doença de Chagas. Memórias do Instituto Oswaldo Cruz 74:285-288, 1976.

2. Brumpt E. Le xénodiagnostic. Aplication au diagnostic de quelques infections parasitaires et en particulier à la Trypanosomose de Chagas. Bulletin de la Société de Pathologie Exotique 7:706-710, 1914. 
Nunes EV, Campos R, Guilherme CS, Tolezano JE, Moreira AAB, Souza HBW, Takiguti CX, Amato-Neto V. $O$ xenodiagnóstico na doença de Chagas: influência do sexo dos triatomíneos. Revista da Sociedade Brasileira de Medicina Tropical 24:245-250, out-dez, 1991

3. Cedillos RA, Hubsch R, Tonn RJ, Escalante MP, Carrasquero B, Liendo H. Comparacion de los metodos de laboratório para examinar xenodiagnóstico. Boletin de la Oficina Sanitária Panamericana 92:49-56, 1982.

4. Cedillos RA, Torrealba JW, Tonn RJ, Mosca W, Ortegón A. El xenodiagnóstico artificial en la enfermedad de Chagas. Boletin de la Oficina Sanitária Panamericana 93:240-249, 1982.

5. Cerisola JA, Rohwedder R, Segura EL, Del Prado CE, Alvarez M, De Martini GJW. El xenodiagnóstico. Instituto Nacional de Diagnóstico e Investigación de la Enfermedad de Chagas Dr. Mário Fatala Chaben, Buenos Aires, 1974.

6. Chiari $\mathrm{E}$, Brener $\mathrm{Z}$. Contribuição ao diagnóstico parasitológico da doença de Chagas na sua fase crônica. Revista do Instituto de Medicina Tropical de São Paulo 8:134-138, 1966.

7. Dias E. Xenodiagnostique appliqué à la trypanosomíase americana. Comptes Rendus de Seances de la Société de Biologie et de ses Filiales 118:187-189, 1934.

8: Dias E. Técnica de xenodiagnóstico na moléstica de Chagas. Memórias do Instituto Oswaldo Cruz 35:335-342, 1940.

9. Espinola HN. Nota sobre diferenças sexuais em formas imatura de Triatominae (Hemíptera, Reduviidae). Revista Brasileira de Biologia 26:263267, 1966.

10. Freitas JLP. Observações sobre o tempo ótimo para o exame de triatomíneos empregados em xenodiagnósticos. Folia Clínica et Biológica 16:180-185, 1950.

11. Galliard H. Recherches aur les reduvidés hématophages Rhodnius et Triatoma. Annales de parasitologia XIII:289-306, 1935.

12. Perlowagora-Szumlewicz A, Cruz HN. Triatominae (Hemíptera, Reduviidae) sex identification in immature forms of vectors of Chagas disease. Revista do Instituto de Medicina
Tropical de São Paulo 14:6-11, 1972.

13. Pifano FC. El diagnóstico parasitológico de la enfermedad de Chagas en fase crônica. Estudio comparativo entre la gota gruesa, el xenodiagnóstico, el hemocultivo y las inoculações experimentales en animales sensibles. Archivos Venezolanos de Patologia Tropical y Parasitologia Médica 2:121-156, 1954.

14. Pifano FC. Evaluación de los procedimientos de laboratório empleados en el diagnóstico de la enfermedad de Chagas. Boletin de la Oficina Sanitária Panamericana 49:563-571, 1960.

15. Salgado AA. Consideraciones sobre metodologia y sensibilidad del xenodiagnóstico. Boletin Chileno de Parasitologia 4:9-13, 1969.

16. Schenone H, Alfaro E, Reyes $H$, Taucher E. Valor del xenodiagnóstico en la infección chagásica crônica. Boletin Chileno de Parasitologia 23:149154, 1968.

17. Siqueira AF. Diagnóstico parasitológico. In: Cançado JR (ed) Doença de Chagas. Imprensa Oficial Estado de Minas Gerais Belo Horizonte p.261-278, 1968.

18. Tolezano JE, Araújo MFL, Chieffi PP, Valentim AM, Ribeiro SS. Variáveis relacionadas ao desenvolvimento de Triatoma infestans Klug, 1834 em condições de laboratório. 2. Efeitos da manipulação na sobrevivência e na fertilidade. Revista do Instituto Adolfo Lutz 44:81-86, 1984.

19. Tolezano JE, Araújo MFL, Ribeiro SS, Ishida MMI. Efeitos do jejum e da temperatura em laboratório na infectividade de triatomíneos por Trypanosoma cruzi. Revista do Instituto Adolfo Lutz 43:25-32, 1983.

20. Tolezano JE, Chieffi PP, Araújo MFL, Valentim AM, Ribeiro SS. Variáveis relacionadas ao desenvolvimento de Triatoma infestans Klug, 1834 em condições de laboratório. 1. Relação entre o repasto sanguíneo e o desenvolvimento. Revista do Instituto Adolfo Lutz 4:73-79, 1984. 